\title{
The effects of nicotine on laser Doppler measures of cochlear blood flow
}

\author{
H.A. Dengerink ${ }^{1}$, J.W. Wright ${ }^{1}$, J.M. Miller ${ }^{2}$ and P. Goodwin ${ }^{3}$ \\ ${ }^{\prime}$ Department of Psychology, Washington State University, Pullman, WA 99164-4830, 'Kresge Hearing Research Institute, University of \\ Michigan, Ann Arbor, MI 48109, and ${ }^{3}$ Department of Otolaryngology, University of Washington, Seattle, WA 98195, U.S.A.
}

(Received 4 April 1985; accepted 7 August 1985)

\begin{abstract}
Anesthetized guinea pigs were given arterial bolus injections of saline or varying nicotine concentrations. Blood flow through the cochlea and skin were measured via laser Doppler and arterial blood pressure via an arterial cannula. Cochlear blood flow increased with low duses of nicotine but decreased with the highest dose, while blood pressure increased and skin flow decreased with all doses of nicotine. Control injections of saline vehicle had only minor and transient effects.
\end{abstract}

cochlear blood flow, arterial blood pressure, nicotine, laser Doppler flowmeter, guinea pig

Nicotine is a cholinomimetic agent and specifically a ganglionic stimulating and blocking agent (Taylor, 1980; Volle and Koelle, 1970). As such it has a variety of neurologically mediated effects on the cardiovascular system. The effect of these nicotine induced cardiovascular changes on perfusion of the cochlear vasculature is not known. There appear, however, to be two basic mechanisms by which nicotine may affect cochlear blood flow (CBF). First, CBF may be altered secondary to systemic changes in cardiovascular parameters. Nicotine elevates heart rate, increases blood pressure and promotes peripheral vasoconstriction (Ague, 1973, 1974; Bobbin and Gondra, 1976; Cellina et al., 1975; Elliott and Thysell, 1968; Erwin, 1971; Myrsten et al., 1972; Nesbitt, 1973). Several authors have reported that CBF varies in proportion to systemic blood pressure (Goodwin et al., 1985; Miller et al., 1983, 1985; Snow and Suga, 1972; Suga and Snow, 1969). Wright et al. (1985) for example, reported that systemic blood pressure and cochlear blood flow both increased as a result of infusing angiotensin, an arterial vasoconstrictor.

A second means by which nicotine may affect cochlear blood flow is via regional or even local mechanisms. Such effects could conceivably occur directly or seondary to catecholamine release. Al- though the relationship to CBF is not known, findings concerning the effects of nicotine on cerebral blood flow may be applicable. Hall (1972) has reported that nicotine elevated cerebral blood flow. He attributed such increases to local vasodilating mechanisms within the brain. More specifically, Vatner et al. (1980) reported that a 0.2 $\mu \mathrm{g} / \mathrm{kg}$ dose of nicotine resulted first in elevated cerebral blood flow and velocity and then in a decrease. They attributed these changes to actions of the carotid chemoreceptors and the sympathetic nervous system. The later decrement in cerebral perfusion, however, was independent of mean arterial pressure which remained elevated, suggesting the possibility of local mechanisms.

Despite the aforementioned indications that nicotine may influence CBF, no previous study, to our knowledge, has directly examined the effect of nicotine on CBF. The purpose of the present investigation was to measure nicotine induced changes in CBF via a laser Doppler flowmeter.

\section{Method}

Subjects

The subjects for this experiment were 9 mature (approximately $1000 \mathrm{~g})$ Hartley strain albino guinea pigs (Charles River). They were maintained 
in group cages in a vivarium approved by the American Association for Laboratory Animal Care, with a $12: 12$ light/dark cycle initiated at $0700 \mathrm{~h}$, and a freely available nutritionally balanced diet. Each was determined to have normal Preyer reflexes. Examination of the middle ear during surgery revealed no evidence of pathology.

\section{Laser Doppler flowmeter}

A Doppler system has recently been developed to measure blood flow using coherent laser light (Powers and Frayer, 1978; Stern, 1975). This technique has been applied to skin and renal cortex and has been shown for these structures to provide a measure which correlates in a linear fashion with blood flow as measured via radioactive xenon clearance and radioactive microspheres (Holloway and Watkins, 1977; Oberg et al., 1979; Stern et al., 1977)

The system uses a helium-neon laser light ( 632.8 $\mathrm{nm}$ ). Moving particles create a spectral shift in the reflected light. In the laser Doppler instrument the reading is proportional to the product of the velocity of the red blood cells (RBCs) within the measurement volume and the volume fraction (number of RBCs) (Nilsson et al., 1980). The product of volume and velocity is referred to as flux.

The Doppler probe is sensitive to reflected light in a volume of $1 \mathrm{~mm}^{3}$ (Bonner et al., 1981). In the skin the depth of penetration is up to $1.5 \mathrm{~mm}$ but has been judged to be effectively $1.0 \mathrm{~mm}$ (Nilsson et al., 1980). It is not known what the effective depth of penetration is in the cochlea but is anticipated to be less than that observed in the less dense skin. Given the measurement volume, the probe placement, and the apparent depth of penetration, it is assumed that the laser Doppler measures blood flow in the following cochlear vessels: capillaries of the spiral ligament including those of the scala vestibuli, the stria vascularis, vessels of the spiral prominence, initial segments of the collecting venules, and perhaps vessels of the basilar membrane and osseous spiral lamina. Several recent investigations suggest that the laser Doppler is a measure which is proportional to cochlear blood flow changes as influenced by catecholamines, angiotensin, carbon monoxide, and local cooling (Goodwin et al., 1985; Miller et al., 1983, 1985; Short et al., 1984; Wright et al., 1985).

\section{Surgical procedures}

Ketamine $(110 \mathrm{mg} / \mathrm{kg})$ and Xylazine (13 $\mathrm{mg} / \mathrm{kg}$ ) were employed as the anesthetic. Supplemental injections (20:1 Ketamine: Xylazine) were employed as needed. A mounting post was secured to the animal's head with stainless-steel screws and dental acrylic. The trachea was cannulated to permit unrestricted breathing. Blood pressure was monitored by a Statham (Model P23Db) strain type pressure gauge, via a femoral artery cannula (Type MRE-040, micro-renathan, Braintree Scientific) which also permitted infusion of saline and nicotine solutions. The catheter was tapered by heating it in vegetable oil $\left(140^{\circ} \mathrm{C}\right)$ for $20 \mathrm{~s}$ and then pulling it to the desired diameter. In this way the catheter could be inserted until the tip was at or near the junction of the femoral and descending aorta arteries.

The bulla was opened via a ventral approach exposing the cochlea. The lateral wall mucosa was gently removed with a cotton pledgette to eliminate mucosal vasculature and to permit approximation of the measurement probe to the bony surface of the lateral wall of the basal turn. In all other respects the middle ear was left intact. The needle probe (O.D. $1.75 \mathrm{~mm}$ ) of the laser Doppler flowmeter (Medpacific, LD-5000) was positioned over the stria vasculature as described previously (Goodwin et al., 1985; Miller et al., 1983; Short et al., 1984; Wright et al., 1985). A second Doppler flowmeter was positioned over a shaved portion of the animal's abdomen to measure skin blood flow. Core temperature was maintained by means of a heating pad placed beneath the animal. The output of both flowmeters and the strain gauge was monitored via a Sanborn polygraph.

\section{Experimental procedures and analyses}

Baseline measurements were obtained for blood pressure, cochlear and abdominal skin blood flow. Repeated observations were made on each of the nine animals. Bolus infusions of $0.25 \mathrm{ml}$ saline (vehicle, $n=5$ ) or nicotine (Sigma Chemicals) solutions at doses of $0.2(n=3), 0.1(n=8), 0.05$ $(n=8)$, or $0.01 \quad(n=6) \mu \mathrm{g} / \mathrm{kg}$ of body weight were administered via the femoral artery cannula. The order of the saline and nicotine doses was random to reduce cumulative effects. Following each infusion all measures were permitted to re- 
turn to the pre-infusion baseline and demonstrate stability for at least $10 \mathrm{~min}$ before subsequent injections.

Each dependent measure (blood pressure. cochlear blood flow and skin blood flow) was quantified at six second intervals beginning $36 \mathrm{~s}$ prior to the onset of infusion and continued until all measures returned to baseline. The 6 measures taken prior to the infusion were averaged to provide an estimate of baseline. All subsequent scores were then calculated as a proportion of that baseline. Adjacent pairs of scores were averaged and plotted in Figs. 1-3. In each of these figures the pre-infusion baseline is equal to a proportion score of 1.00 .

Two additional measures were also calculated employing the $6 \mathrm{~s}$ scores. These were the score which represented the maximum deviation from baseline (either positive or negative) expressed as a proportion of baseline and the number of seconds until the first return to baseline for $12 \mathrm{~s}$ or more. These latter two measures were used for statistical analyses. These measures evidenced considerable heterogeneity of variance. The large differences in variance for the various conditions precluded meeting the assumptions for many statistical tests including analysis of variance and Duncan's Multiple Range test. Consequently $t$-tests were employed to assess the significance of the differences between treatments. It should be noted, however, that similar statistical results were obtained when Duncan's Multiple Range test was employed.

\section{Results}

The mean arterial pressure during baseline averaged $48 \pm 3.16$ (S.E.) $\mathrm{mmHg}$. Fig. 1 displays the average blood pressure (expressed as proportion of baseline) for each condition as a function of time after injection. Saline had a minor elevating effect on blood pressure while nicotine infusions resulted in major elevations in blood pressure above baseline. The largest of these increases was observed for the highest dose $(0.2 \mu \mathrm{g})$ and peaked between 36 and $60 \mathrm{~s}$ after the injection. The other doses of nicotine, while producing greater elevations than saline, and smaller effects than the highest dose did not appear to differ from each other. Statistical analysis, however, failed to

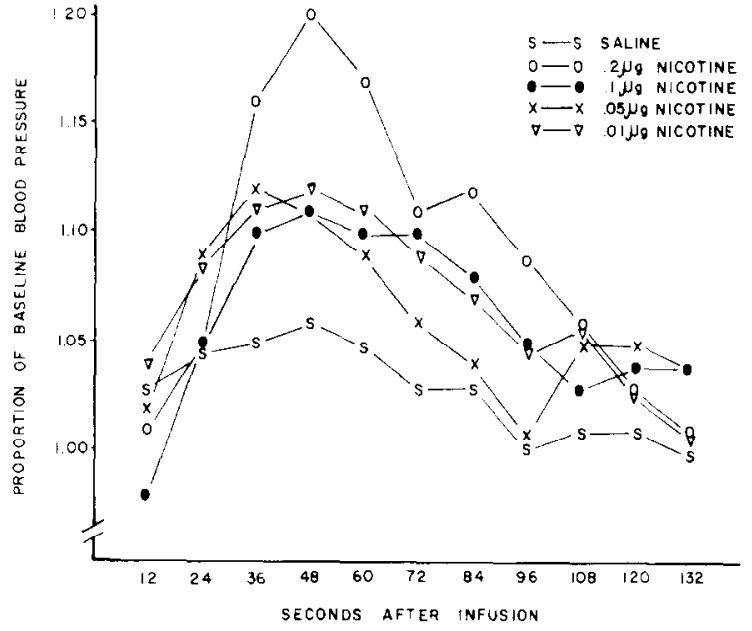

Fig. 1. Mean blood pressure plotted as proportion of baseline for saline and nicotine groups as a function of time after infusion.

indicate significant differences between the saline and any of the nicotine treatments in either maximum change or in duration.

Fig. 2 displays the average skin blood flow for each of the treatment conditions. Skin blood flow varied slightly from baseline in the saline condition. In the nicotine conditions, skin blood flow decreased relative to baseline. Further, the magnitude of the effect increased with increasing doses. The maximum change from baseline for the saline, $0.01,0.05,0.1$ and $0.2 \mu \mathrm{g}$ nicotine conditions

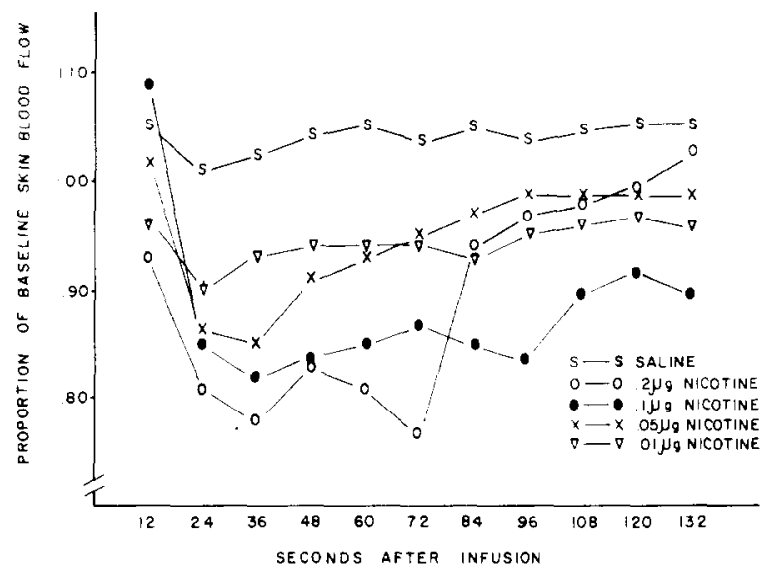

Fig. 2. Skin blood flow plotted as proportion of baseline for saline and nicotine groups as a function of time after infusion. 
averaged ( \pm S.E.) $1.08(0.02), 0.80(0.04), 0.82$ $(0.08), 0.79(0.08)$ and $0.76(0.10)$, respectively. The number of seconds until the first return to baseline averaged $( \pm$ S.E.) $22.8(5.8), 40.8(6.9), 48.5(11.11)$, $69.8(10.7)$ and $70.0(17.7)$ in the saline $0.01,0.05$, 0.1 and $0.2 \mu \mathrm{g}$ nicotine conditions, respectively. Statistical analyses indicated that the saline condition was significantly ( $P<0.05$ or less) different from each of the nicotine conditions both in the maximum effect and in the duration of the effect. Further, the $0.2 \mu \mathrm{g}$ condition required significantly longer time for the first return to baseline than did the remaining nicotine conditions.

Fig. 3 displays the average cochlear blood flow in proportion of baseline for each of the treatment conditions. Relatively minor changes were observed in the saline condition. The two lowest doses of nicotine resulted in the greatest elevation in cochlear blood flow and relatively slow returns to baseline. The $0.1 \mu \mathrm{g}$ dose of nicotine resulted in an elevation comparable to that of the lower doses and appeared to return to baseline rapidly. In contrast the highest dose of nicotine $(0.2 \mu \mathrm{g}) \mathrm{re}$ sulted in a decrement in cochlear blood flow which was slow to occur and which was slow to return to baseline. The average maximum CBF ( \pm S.E.) was $1.05(0.01), 1.17(0.02), 1.20(0.07), 1.08(0.10)$ and $0.87(0.19)$ for the saline, $0.01,0.05,0.1$ and $0.2 \mu \mathrm{g}$ nicotine conditions, respectively. The average number of seconds $( \pm$ S.E.) until returning to

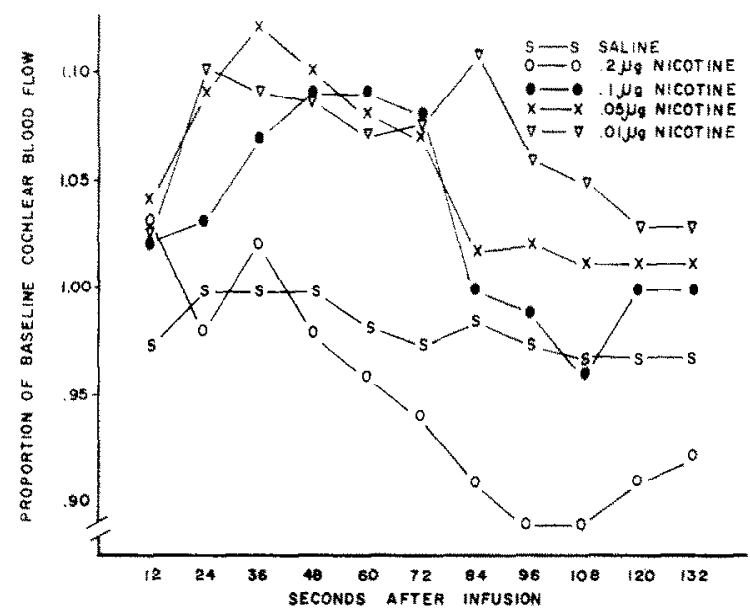

Fig. 3. Cochlear blood flow plotted as proportion of baseline for saline and nicotine groups as a function of time after infusion. baseline was $32.6(13.7), 55.0(7.8) .63 .0(10.9)$, $61.5(8.1)$ and $92.0(25.7)$ for the saline, $0.01,0.05$, 0.1 and $0.2 \mu \mathrm{g}$ nicotine conditions, respectively. The statistical analysis indicated that only the 0.01 and $0.05 \mu \mathrm{g}$ nicotine conditions resulted in deviations from baseline which were significantly $(P<$ $0.05)$ greater than those for the saline condition. Further the maximum change observed in the 0.2 $\mu \mathrm{g}$ nicotine condition was significantly different from the maximum change observed in the 0.05 and $0.01 \mu \mathrm{g}$ nicotine conditions but not different from the saline condition. The duration of the change was significantly greater in the $0.05,0.1$ and $0.2 \mu \mathrm{g}$ nicotine conditions than in the saline condition.

\section{Discussion}

The major observation of the current study was that moderate doses of nicotine elevate CBF to a greater extent than does saline and that that elevation lasts longer than the changes induced by saline. The elevations in CBF noted in the moderate doses may be attributable to systemic changes. That is, the changes in CBF noted in these conditions may be passive ones which are secondary to increased systemic blood pressure and cutaneous vasoconstriction. A similar conclusion has been reached by others (Goodwin et al, 1985; Miller et al., 1983, 1985; Snow and Suga, 1972; Suga and Snow, 1969; Wright et al., 1985). In the current results, however, blood pressure changes in response to nicotine were not statistically significant while changes in skin and cochlear blood flow were. This observation detracts from the possibility of explaining CBF changes as being secondary to systemic blood pressure changes.

Furthermore, the changes in CBF observed in the higher dose conditions of the current experiment may require another explanation. The failure of the $0.1 \mu \mathrm{g}$ dose to elevate CBF to a level that was significantly greater than that observed in the saline condition, and the negative change in CBF observed for the $0.2 \mu \mathrm{g}$ dose are not consistent with the changes observed in cutaneous blood flow or the direction of the changes in blood pressure. An explanation other than passive responsiveness secondary to systemic blood pressure is required for these higher dose effects. One possible ex- 
planation relates to the ganglionic stimulating and blocking effect of nicotine (Taylor, 1980; Volle and Koelle, 1970). Since nicotine has both effects it is possible that the smaller change in the $0.1 \mu \mathrm{g}$ condition and the negative change in the $0.2 \mu \mathrm{g}$ condition represent the protracted blocking effect of nicotine on sympathetic ganglia. That is, the ganglionic receptors may have been saturated by the higher doses of nicotine which may then have blocked further effects of nicotine and inhibited normal mechanisms by which CBF is maintained.

A second possible explanation for the unanticipated effects of the higher doses is that these findings may reflect some regional or local control of CBF independent of systemic changes. Such changes could reflect direct effects of nicotine on CBF or effects secondary to catecholamine release which in some manner control CBF. Previous authors have suggested that catecholamines do have a local vasoconstrictive effect within the ear (Muchnik et al., 1983). If CBF and cerebral blood flow are similarly controlled, then it is interesting to note that the dose which produced a decrement in CBF $(0.2 \mu \mathrm{g})$ is the same dose for which Vatner et al. (1980) observed an initial increase and then a decrease in cerebral blood flow in dogs.

While the current findings indicate that low to moderate doses of nicotine elevate cochlear blood flow, some cautions are warranted. The laser Doppler measures of cochlear blood flow require an anesthetized preparation. The blood pressures noted in the current results were somewhat lower than may be expected for normal resting blood pressures. The observed average baseline blood pressure was $48 \mathrm{mmHg}$. Other authors have reported that resting awake blood pressures in guinea pigs average $50-70 \mathrm{mmHg}$ (Marshall and Hanna, 1956; Muchnik et al., 1980). It is not known what effect anesthesia or reduced blood pressure may have on the current measures of cochlear blood flow.

It should also be pointed out that the Doppler flowmeter provides a measure of flux: the product of red cell volume fraction and their mean velocity. Thus, while the current findings indicate that low to moderate doses of nicotine elevate CBF, it is not known whether that elevation represents high blood cell velocity, greater volume fraction or both. Clarification of the difference may lead to clarification in the mechanism by which nicotine elevates CBF.

\section{References}

Ague, C. (1973): Smoking patterns, nicotine intake at different times of day and changes in two cardiovascular variables while smoking cigarettes. Psychopharmacologia 30 , $135-144$.

Ague, C. (1974): Cardiovascular variables, skin conductance and time estimation: Changes after the administration of small doses of nicotine. Psychopharmacologia 37, 109-125.

Bobbin, R.P. and Gondra, M.I. (1976): Effect of nicotine on cochlear function and noise-induced hair cell loss. Ann. Otol. $85,247-254$.

Bonner, R.F., Clem, T.R., Bowen, P.D. and Bowman, R.L. (1981): Laser Doppler real-time monitor of pulsatile and mean blood flow in tissue microcirculation. In: Scattering Techniques Applied to Supra Molecular and Non-equilibrium Systems (NATO ASI Senses B, Vol. 73), pp. 685-710, Editors: S.H. Chow, B. Chu and R. Nossal. Plenum Press, New York.

Cellina, G.U.. Honour, A.J. and Littler, W.A. (1975): Direct arterial pressure, heart rate. and electrocardiogram during cigarette smoking in unrestricted patients. Am. Heart J. 89 , $18-25$.

Elliott, R. and Thysell, R. (1968): A note on smoking and heart rate. Psychophysiology 5, 280-283.

Erwin, C.W. (1971): Cardiac rate responses to cigarette smoking: a study utilizing radiotelemetry. Psychophysiology 8 . $75-81$.

Goodwin, P., Miller, J.M., Dengerink, H.A., Wright, J.W. and Axelsson, A. (1985): The laser Doppler: A non-invasive measure of cochlear blood flow. Acta-Otolaryngol. (in press).

Hall, G.H. (1972): Effects of nicotine, carbon monoxide and tobacco smoke on regional blood flow in the cerebral cortex. Eur. J. Pharmacol. 19, 385-388.

Holloway, G.A. and Watkins, D.W. (1977): Laser Doppler measurement of cutaneous blood flow. J. Invest. Dermatol. $69,306$.

Marshall, L.H. and Hanna, C.H. (1956): Direct measurement of arterial blood pressure in the guinea pig. Proc. Soc. Exp. Biol. Med. 92, 31-32.

Miller, J.M., Marks, N. and Goodwin, P.C. (1983): Laser Doppler measurement of cochlear blood flow. Hearing Res. 11. 385 .

Miller, J.M., Goodwin, P. and Marks, N. (1985): Inner ear blood flow using a laser Doppler system. Arch. Otolaryngol. (in press).

Muchnik, C., Hildesheimer, M. and Rubenstein, M. (1980): Effect of emotional stress on hearing. Arch. Otorhinolaryngol. 228, 295-298.

Muchnik, C., Hildesheimer, M., Nebel, L. and Rubenstein, M. (1983): Influence of catecholamine on cochlear action potentials. Arch. Otolaryngol. 109, 530-532.

Myrsten, A., Post, B., Frankenhaeuser, M. and Johansson, G. (1972): Changes in behavioral and physiological activation induced by cigarette smoking in habitual smokers. Psychopharmacologia $27,305-312$. 
Nesbitt, P.D. (1973): Smoking, physiological arousal and emotional response. Psychophysiology 25, 137-144.

Nilsson, G.E., Tenland, T. and Oberg, P.A. (1980): Evaluation of a laser Doppler flowmeter for measurement of tissue blood flow. IEEE Trans. Biomed. Eng. 27, 12.

Oberg, P.A., Nilsson, G.E., Tenland, T., Homstrom, A. and Lewis, D.H. (1979): Use of a new laser Doppler flowmeter for measurement of capillary blood flow in skeletal muscle after bullet wounding. Acta Chir. Scand. Suppl. 489, 145.

Powers, E.W. and Frayer, W.W. (1978): Laser Doppler measurement of blood flow in microcirculation. Plastic Reconstructive Surg. 61, 250.

Short, S., Goodwin, P.C. and Miller, J.M. (1984): Measuring cochlear blood flow using laser Doppler Spectroscopy. Paper presented at the Meeting of the Association for Research in Otolaryngology, St. Petersburg, FL.

Snow, J.B. and Suga, G. (1972): Control of cochlear blood flow. In: Vascular Disorders and Hearing Defects, pp. 167-183. Editor: J.D. De Lorenzo. University Park Press, Baltimore.

Stern, M.D. (1975): In vivo evaluation of microcirculation by coherent light scattering. Nature (London) 254, 56-58.
Stern, M.D., Lappe, D.L., Bowen, P.D., Chimosky, J.E., Holloway, G.A., Geiser, H.R. and Bowen, R.L. (1977): Continuous measurement of tissue blood flow by laser Doppler spectroscopy. Am. J. Physiol. 232, H441.

Suga, F. and Snow, J.B. (1969): Cochlear blood flow in response to vasodilating drugs and some related agents. Laryngoscope 79, 1956-1979.

Taylor, P. (1980): Ganglionic stimulating and blocking agents. In: The Pharmacological Basis of Therapeutics, pp. 211-219. Editors: L. Goodman and A. Gillman. Macmillan, New York.

Vatner, S.F., Priano, L.L., Rutherford, J.D. and Manders, T. (1980): Sympathetic regulation of the cerebral circulation by the carotid chemoreceptor reflex. Am. J. Physiol. 80 , H594-H598.

Volle, R.L. and Koelle, G.B. (1970): Ganglionic stimulating and blocking agents. In: The Pharmacological Basis of Therapeutics, pp. 585-595. Editors: L. Goodman and A Gillman. Macmillan, New York.

Wright, J.W., Dengerink, H.A., Miller, J.M. and Goodwin, P.C. (1985): Potential role of angiotensin II in noise-induced increases in inner ear blood flow. Hearing Res. 17, 41-46. 